\title{
Alterations in the Rate of Limb Movement Using a Lower Body Positive Pressure Treadmill Do Not Influence Respiratory Rate or Phase III Ventilation
}

\author{
Michael J. Buono, Marissa Burnsed-Torres, Bethany Hess, Kristine Lopez, Catherine Ortiz, \\ Ariel Girodo, Karen Lolli, Brett Bloom, David Bailey, and Fred W. Kolkhorst
}

Department of Biology, School of Exercise and Nutritional Sciences, San Diego State University, San Diego, CA 92182-7251, USA

Correspondence should be addressed to Michael J. Buono; mbuono@mail.sdsu.edu

Received 7 November 2014; Revised 16 December 2014; Accepted 22 December 2014

Academic Editor: Leonardo F. Ferreira

Copyright (C) 2015 Michael J. Buono et al. This is an open access article distributed under the Creative Commons Attribution License, which permits unrestricted use, distribution, and reproduction in any medium, provided the original work is properly cited.

\begin{abstract}
The purpose of this study was to determine the effect of alterations in rate of limb movement on Phase III ventilation during exercise, independent of metabolic rate, gait style, and treadmill incline. Subjects completed five submaximal exercise bouts on a lower body positive pressure treadmill (AlterG P 200). The percent body weight for the five exercise bouts was 100, 87, 75, 63, and 50\% and each was matched for carbon dioxide production $\left(V_{\mathrm{CO}_{2}}\right)$. Naturally, to match the $V_{\mathrm{CO}_{2}}$ while reducing the body weight up to $50 \%$ of normal required a significant increase in the treadmill speed from $3.0 \pm 0.1$ to $4.1 \pm 0.2 \mathrm{mph}$, which resulted in a significant $(P<0.05)$ increase in the mean step frequency (steps per minute) from $118 \pm 10$ at $3 \mathrm{mph}$ (i.e., $100 \%$ of body weight) to $133 \pm 6$ at $4.1 \mathrm{mph}$ (i.e., $50 \%$ of body weight). The most important finding was that significant increases in step frequency did not significantly alter minute ventilation or respiratory rate. Such results do not support an important role for the rate of limb movement in Phase III ventilation during submaximal exercise, when metabolic rate, gait style, and treadmill incline are controlled.
\end{abstract}

\section{Introduction}

Even after a century of investigation the control mechanisms responsible for the hyperpnea seen during steady state exercise (i.e., Phase III) are still under debate. One area that is particularly controversial is the role that limb movement has in exercise hyperpnea $[1,2]$. A common experimental design used in the past to investigate the topic has been to alter the rate of limb movement during inclined treadmill walking versus horizontal treadmill running, while matching the metabolic cost of the two gait styles [3-7].

Collectively a dichotomous situation exists in the literature with some of these studies supporting the importance of limb movement frequency in exercise hyperpnea $[5,7,8]$ while others do not $[3,4,6,9]$. One common potential concern with the methodological design used in these past investigations is that two different gait styles (i.e., inclined walking versus horizontal running) were used to alter the frequency of limb movement.
Three lines of reasoning support the contention that controlling gait style may be important when examining exercise hyperpnea. First, studies have shown that both gait style and treadmill incline significantly affect the pattern of muscle recruitment, Achilles tendon stretch, and muscle strain in the lower limbs during exercise [10-12], all of which may alter exercise hyperpnea. Second, it is known that subjects rate uphill walking as being more difficult when compared to horizontal running at similar metabolic rates and that "perception of effort" during exercise can clearly alter exercise hyperpnea $[13,14]$. Lastly, it has been suggested that inclined walking alters the vestibular-originated drive to breath compared to horizontal running due to differences in head motion between the two gait styles [1].

In an attempt to reduce the potential problems associated with past studies that have used two different gait styles, we have incorporated the recently developed lower body positive pressure treadmill $[15,16]$. Specifically, the research design used in the current study allowed us to reduce the body 
TABLE 1: Mean $( \pm S D)$ physiological responses to reduced body weight exercise.

\begin{tabular}{|c|c|c|c|c|c|c|}
\hline Body weight (\%) & $\begin{array}{l}\text { Walking speed } \\
(\mathrm{mph})\end{array}$ & $\begin{array}{c}V_{\mathrm{O}_{2}} \\
(\mathrm{~L} / \mathrm{min})\end{array}$ & $\begin{array}{c}V_{\mathrm{CO}_{2}} \\
(\mathrm{~L} / \mathrm{min})\end{array}$ & $\begin{array}{l}\text { Frequency } \\
\text { (steps/min) }\end{array}$ & $\begin{array}{c}V_{\mathrm{E}} \\
(\mathrm{L} / \mathrm{min})\end{array}$ & $\begin{array}{c}\text { Respiratory rate } \\
\text { (breaths/min) }\end{array}$ \\
\hline $100 \%$ & $3.0 \pm 0.1$ & $1.23 \pm 0.36$ & $1.04 \pm 0.27$ & $118 \pm 10$ & $33.0 \pm 8.4$ & $26 \pm 4$ \\
\hline $87 \%$ & $3.3 \pm 0.2$ & $1.20 \pm 0.34$ & $1.04 \pm 0.25$ & $124 \pm 9^{*}$ & $32.1 \pm 8.0$ & $25 \pm 4$ \\
\hline $75 \%$ & $3.6 \pm 0.2$ & $1.23 \pm 0.33$ & $1.05 \pm 0.24$ & $129 \pm 8^{* \#}$ & $32.9 \pm 8.1$ & $26 \pm 5$ \\
\hline $63 \%$ & $3.9 \pm 0.1$ & $1.20 \pm 0.33$ & $1.04 \pm 0.26$ & $133 \pm 6^{* \#}$ & $33.4 \pm 8.4$ & $27 \pm 6$ \\
\hline $50 \%$ & $4.1 \pm 0.2$ & $1.19 \pm 0.33$ & $1.05 \pm 0.25$ & $133 \pm 6^{* \# \bullet}$ & $33.1 \pm 8.0$ & $26 \pm 5$ \\
\hline
\end{tabular}

${ }^{*}$ indicates that value is significantly $(P<0.05)$ different than the $100 \% \mathrm{BW}$ value, ${ }^{\#}$ indicates that value is significantly $(P<0.05)$ different than the $87 \% \mathrm{BW}$ value, and indicates that value is significantly $(P<0.05)$ different than the $75 \%$ BW value.

weight of walking subjects while simultaneously increasing the speed, thus matching the metabolic cost of each workload. For example, walking at $3 \mathrm{mph}$ at $100 \%$ body weight resulted in the same oxygen uptake and carbon dioxide production as walking at $4.1 \mathrm{mph}$ at $50 \%$ of the body weight. Therefore, in the current study we could independently alter the stepping rate and thus the rate of limb movement while allowing the subjects to only use one gait style. Such a methodological design alleviates some of the potential concerns associated with past studies. It was hypothesized that the rate of limb movement would not significantly affect Phase III ventilation during submaximal exercise, when metabolic rate, gait style, and treadmill incline are controlled.

\section{Methods}

2.1. Subjects. The subjects for this study were 10 healthy volunteers ( 4 males and 6 females). The group had mean $( \pm S D)$ age, height, and weight of $31.3 \pm 14.2 \mathrm{y}, 169.1 \pm 14.0 \mathrm{~cm}$, and $67.3 \pm 16.8 \mathrm{~kg}$, respectively. The study was approved by the San Diego State University IRB and signed informed consent was obtained.

2.2. Experimental Procedures. Each subject completed five submaximal exercise bouts on a lower body positive pressure treadmill (AlterG P 200). This device consists of a treadmill enclosed within a waist-high plastic chamber that has clear sides for viewing the subject in contact with the treadmill belt. A seal is achieved between the subject and the chamber through the use of a neoprene skirt fitted around the subject's waist that fastens over the opening of the plastic chamber. For unweighting, the pressure inside the chamber was elevated above the external, ambient pressure using an air compressor, with body weight being measured by a force transducer under the treadmill belt. The pressure differential caused an axial buoyant force that lifts the subject off the treadmill belt until the desired percent reduction in body weight is achieved. The percent body weight for the five exercise bouts was 100, $87,75,63$, and $50 \%$ and the order was randomly assigned to each subject. Each exercise bout was between 5 and $8 \mathrm{~min}$ in duration as the subject walked at a $5 \%$ incline.

Oxygen uptake $\left(V_{\mathrm{O}_{2}}\right)$, carbon dioxide production $\left(V_{\mathrm{CO}_{2}}\right)$, minute ventilation $\left(V_{\mathrm{E}}\right)^{2}$, and respiratory rate were measured every minute during the exercise bouts via a low resistance one-way valve attached to a calibrated metabolic cart (TrueOne, ParvoMedics, Sandy, UT) which used a heated pneumotach (Hans Rudolph, Kansas City, MO) to measure expired ventilation. The pneumotach was calibrated before testing using a $3 \mathrm{~L}$ syringe. The treadmill speed was adjusted for each subject to match the $V_{\mathrm{CO}_{2}}$ during the five exercise bouts. Two consecutive $V_{\mathrm{CO}_{2}}$ readings within $0.1 \mathrm{~L} / \mathrm{min}$ were considered as evidence that steady state values were obtained during each exercise bout, and the ventilatory and metabolic data collected for those two minutes were averaged for the recorded value. Step frequencies were counted by two investigators using a stopwatch during each exercise bout and were averaged. Repeated measures ANOVA and Tukey's post hoc tests were used to compare the data obtained during the 5 different body weight conditions. Significance was set at the $P<0.05$ level.

\section{Results}

The mean $( \pm S D)$ metabolic and ventilatory data collected for the 5 exercise bouts are presented in Table 1 . As can be seen, we were able to match the metabolic rate as evidenced by the fact that the $V_{\mathrm{O}_{2}}$ and $V_{\mathrm{CO}_{2}}$ were essentially identical and not significantly different for the five exercise trials. Specifically, the mean $V_{\mathrm{CO}_{2}}$ ranged between 1.04 and $1.05 \mathrm{~L} / \mathrm{min}$ for all five conditions. To match the $V_{\mathrm{CO}_{2}}$ while reducing the body weight up to $50 \%$ of normal required a significant increase in the treadmill speed from $3.0 \pm 0.1$ to $4.1 \pm 0.2 \mathrm{mph}$. The increased treadmill speed resulted in a significant increase in the mean step frequency from $118 \pm 10$ steps per min at $3 \mathrm{mph}$ (i.e., $100 \%$ of body weight) to $133 \pm 6$ at $4.1 \mathrm{mph}$ (i.e., $50 \%$ of body weight).

The major finding of the current study was that increased step frequency did not alter $V_{\mathrm{E}}$ or respiratory rate. Specifically, mean $V_{\mathrm{E}}$ ranged between 32.1 and $33.4 \mathrm{~L} / \mathrm{min}$ for the five different body weight conditions. Similarly, the mean respiratory rate was not significantly different for the five conditions, ranging between $25 \pm 4$ and $27 \pm 6$ breaths per minute for all five trials.

\section{Discussion}

It is clear that exercise hyperpnea likely requires multiple redundant control mechanisms, including various neural and humoral elements. The current study examined only one 
specific component, namely, the role of limb movement frequency in Phase III ventilation during rhythmic exercise [17]. The importance of limb movement frequency in ventilation during exercise is controversial and past investigators have used numerous different research designs to investigate the response.

One commonly used experimental design has been to alter the rate of limb movement during exercise while keeping the metabolic rate constant. To accomplish this, many investigators have compared the ventilatory responses obtained during inclined treadmill walking versus horizontal treadmill running, while matching the metabolic cost of the two gait styles. Collectively, some of these studies support the importance of the rate of limb movement in exercise hyperpnea [5, $7,8,18]$ while others do not $[3,6]$. One potential concern with the methodological design used in those past investigations is that two different gait styles (i.e., inclined walking versus horizontal running) were used, which may alter the afferent feedback, independent of the rate of limb movement $[4,10-$ 12].

In an attempt to reduce the potential problems associated with past studies that have used two different gait styles, we used a lower body positive pressure treadmill $[15,16]$. Specifically, the research design incorporated in the current study allowed us to reduce the body weight of walking subjects while simultaneously increasing the speed, thus matching the metabolic rate of each workload. For example, walking at $3 \mathrm{mph}$ at $100 \%$ body weight resulted in the same oxygen uptake and carbon dioxide production as walking at $4.1 \mathrm{mph}$ at $50 \%$ of the body weight. Therefore, in the current study we could independently alter the stepping rate and thus the rate of limb movement while allowing the subjects to only use one gait style at the same treadmill incline. Such a methodological design alleviates some of the concerns associated with past studies.

The results of the current study do not support an important role for limb movement frequency during Phase III ventilation, as evidenced by the fact that neither mean $V_{\mathrm{E}}$ nor respiratory rate was significantly different during the five different body weight conditions. Such results agree with the findings of Berry et al. [3] and McMurray and Smith [6] but are in conflict with others $[5,7,8,18]$. A logical question is the following: "Why is there such a discrepancy in the literature concerning the role of limb movement frequency in exercise hyperpnea?" We believe that the answer may lie in which phase (i.e., I, II, or III) of the ventilatory response is examined. For example, Berry et al. [3] were careful not to measure the ventilatory responses during inclined treadmill walking versus horizontal running until the subjects had reached the steady state Phase III level. This agrees with our methodology in that ventilatory parameters were not collected in the current study until the subject had two consecutive minutes of $V_{\mathrm{CO}_{2}}$ within $0.1 \mathrm{~L} / \mathrm{min}$ of each other. Conversely, closer examination of past studies that have found limb movement frequency to significantly affect exercise hyperpnea reveals that their subjects were not in Phase III ventilation but rather most likely in Phase I or II. For example, Wells et al. [7] used sinusoidal changes in treadmill speed and grade to test the hypothesis that limb movement frequency is a significant determinant of exercise hyperpnea. Their findings suggested that changes in limb movement frequency did significantly influence exercise hyperpnea. However, during sinusoidal submaximal exercise the $V_{\mathrm{E}}$ responses are likely most comparable to Phase II, not Phase III, responses to a step change in exercise [2]. Interestingly, when they measured Phase III ventilatory responses during steady state inclined treadmill walking versus horizontal treadmill running at matched metabolic rates, they reported essentially identical mean $V_{\mathrm{E}}$ for the two gait styles (29.3 versus $30.4 \mathrm{~L} / \mathrm{min}$, resp.), which agrees with the current findings. Likewise, Hanson et al. [8] reported a significant $10-25 \%$ increase in $V_{\mathrm{E}}$ during horizontal treadmill running compared to inclined treadmill walking during high intensity exercise $\left(\geq 75 \%\right.$ maximal $\left.V_{\mathrm{O}_{2}}\right)$. However, it is unlikely that at such high exercise intensities Phase III ventilatory responses were obtained [19]. Incidentally, the $V_{\mathrm{E}}$ responses during the two gait styles were identical during lower intensity exercise $\left(\leq 60 \%\right.$ maximal $\left.V_{\mathrm{O}_{2}}\right)$, during which Phase III steady state ventilatory responses were most likely attained. The results of the current study, coupled with past findings, suggest that frequency of limb movement may be important during Phases I and II exercise hyperpnea; however, its role is attenuated if data is collected after the subject reaches Phase III $[3,7,8]$.

In conclusion, this is the first study in the literature that has used a lower body positive pressure treadmill to alter the stepping frequency during treadmill walking while matching the $V_{\mathrm{CO}_{2}}$. The results of the current study suggest that frequency of limb movement is not important during Phase III exercise hyperpnea, when $V_{\mathrm{CO}_{2}}$, gait style, and treadmill incline are controlled.

\section{Conflict of Interests}

The authors declare that there is no conflict of interests regarding the publication of this paper.

\section{References}

[1] H. J. Bell, "Respiratory control at exercise onset: an integrated systems perspective," Respiratory Physiology and Neurobiology, vol. 152, no. 1, pp. 1-15, 2006.

[2] H. V. Forster, P. Haouzi, and J. A. Dempsey, "Control of breathing during exercise," Comprehensive Physiology, vol. 2, no. 1, pp. 743-777, 2012.

[3] M. J. Berry, D. W. Bacharach, and T. Moritani, "Stride frequency and ventilation at constant carbon dioxide output," British Journal of Sports Medicine, vol. 19, no. 4, pp. 210-213, 1985.

[4] M. J. Berry, C. J. Dunn, C. L. Pittman, W. C. Kerr, and N. E. Adair, "Increased ventilation in runners during running as compared to walking at similar metabolic rates," European Journal of Applied Physiology and Occupational Physiology, vol. 73, no. 3-4, pp. 245-250, 1996.

[5] R. G. McMurray and S. W. Ahlborn, "Respiratory responses to running and walking at the same metabolic rate," Respiration Physiology, vol. 47, no. 2, pp. 257-265, 1982.

[6] R. G. McMurray and L. G. Smith, "Ventilatory responses when altering stride frequency at a constant oxygen uptake," Respiration Physiology, vol. 62, no. 1, pp. 117-124, 1985. 
[7] G. D. Wells, T. Diep, and J. Duffin, "The ventilatory response to sine wave variation in exercise loads and limb movement frequency," Respiratory Physiology and Neurobiology, vol. 158, no. 1, pp. 45-50, 2007.

[8] P. Hanson, A. Claremont, J. Dempsey, and W. Reddan, "Determinants and consequences of ventilatory responses to competitive endurance running," Journal of Applied Physiology Respiratory Environmental and Exercise Physiology, vol. 52, no. 3, pp. 615-623, 1982.

[9] J. D. S. Kay, E. S. Petersen, and H. Vejby Christensen, "Breathing in man during steady state exercise on the bicycle at two pedalling frequencies, and during treadmill walking," Journal of Physiology, vol. 251, no. 3, pp. 645-656, 1975.

[10] G. B. Gillis and A. A. Biewener, "Effects of surface grade on proximal hindlimb muscle strain and activation during rat locomotion," Journal of Applied Physiology, vol. 93, no. 5, pp. 1731-1743, 2002.

[11] G. A. Lichtwark and A. M. Wilson, "Interactions between the human gastrocnemius muscle and the Achilles tendon during incline, level and decline locomotion," Journal of Experimental Biology, vol. 209, no. 21, pp. 4379-4388, 2006.

[12] C. M. Wall-Scheffler, E. Chumanov, K. Steudel-Numbers, and B. Heiderscheit, "Electromyography activity across gait and incline: the impact of muscular activity on human morphology," American Journal of Physical Anthropology, vol. 143, no. 4, pp. 601-611, 2010.

[13] M. Amann, G. M. Blain, L. T. Proctor, J. J. Sebranek, D. F. Pegelow, and J. A. Dempsey, "Group III and IV muscle afferents contribute to ventilatory and cardiovascular response to rhythmic exercise in humans," Journal of Applied Physiology, vol. 109, no. 4, pp. 966-976, 2010.

[14] M. J. Berry, A. S. Weyrich, R. A. Robergs, K. M. Krause, and C. P. Ingalls, "Ratings of perceived exertion in individuals with varying fitness levels during walking and running," European Journal of Applied Physiology and Occupational Physiology, vol. 58, no. 5, pp. 494-499, 1989.

[15] A. M. Grabowski, "Metabolic and biomechanical effects of velocity and weight support using a lower-body positive pressure device during walking," Archives of Physical Medicine and Rehabilitation, vol. 91, no. 6, pp. 951-957, 2010.

[16] M. D. Hoffman and H. E. Donaghe, "Physiological responses to body weight-supported treadmill exercise in healthy adults," Archives of Physical Medicine and Rehabilitation, vol. 92, no. 6, pp. 960-966, 2011.

[17] S. A. Ward, "Control of the exercise hyperpnoea in humans: a modeling perspective," Respiration Physiology, vol. 122, no. 2-3, pp. 149-166, 2000.

[18] K. Casey, J. Duffin, C. J. Kelsey, and G. V. McAvoy, "The effect of treadmill speed on ventilation at the start of exercise in man," Journal of Physiology, vol. 391, pp. 13-24, 1987.

[19] S. M. Marcora, A. Bosio, and H. M. de Morree, "Locomotor muscle fatigue increases cardiorespiratory responses and reduces performance during intense cycling exercise independently from metabolic stress," The American Journal of Physiology-Regulatory Integrative and Comparative Physiology, vol. 294, no. 3, pp. R874-R883, 2008. 

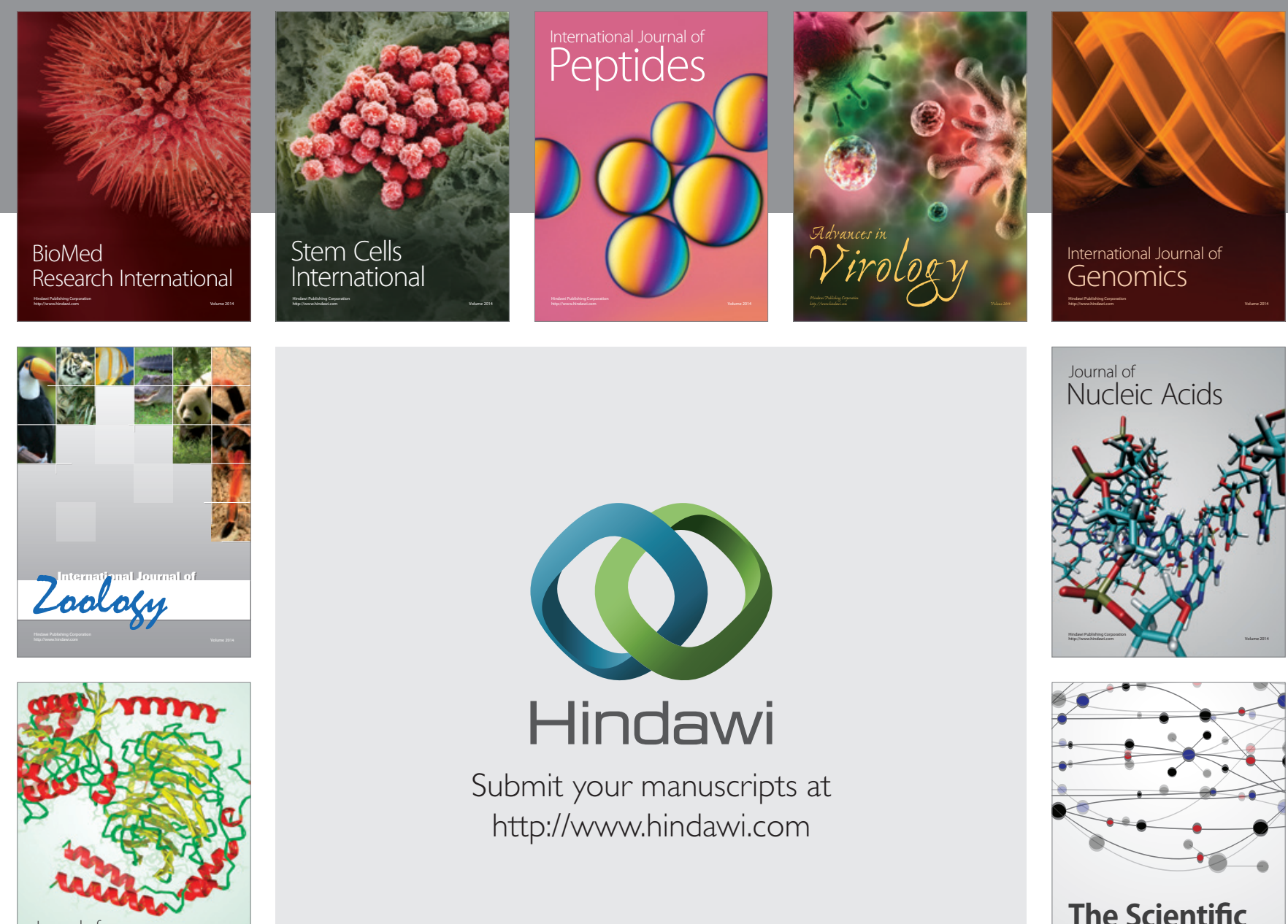

Submit your manuscripts at

http://www.hindawi.com

Journal of
Signal Transduction
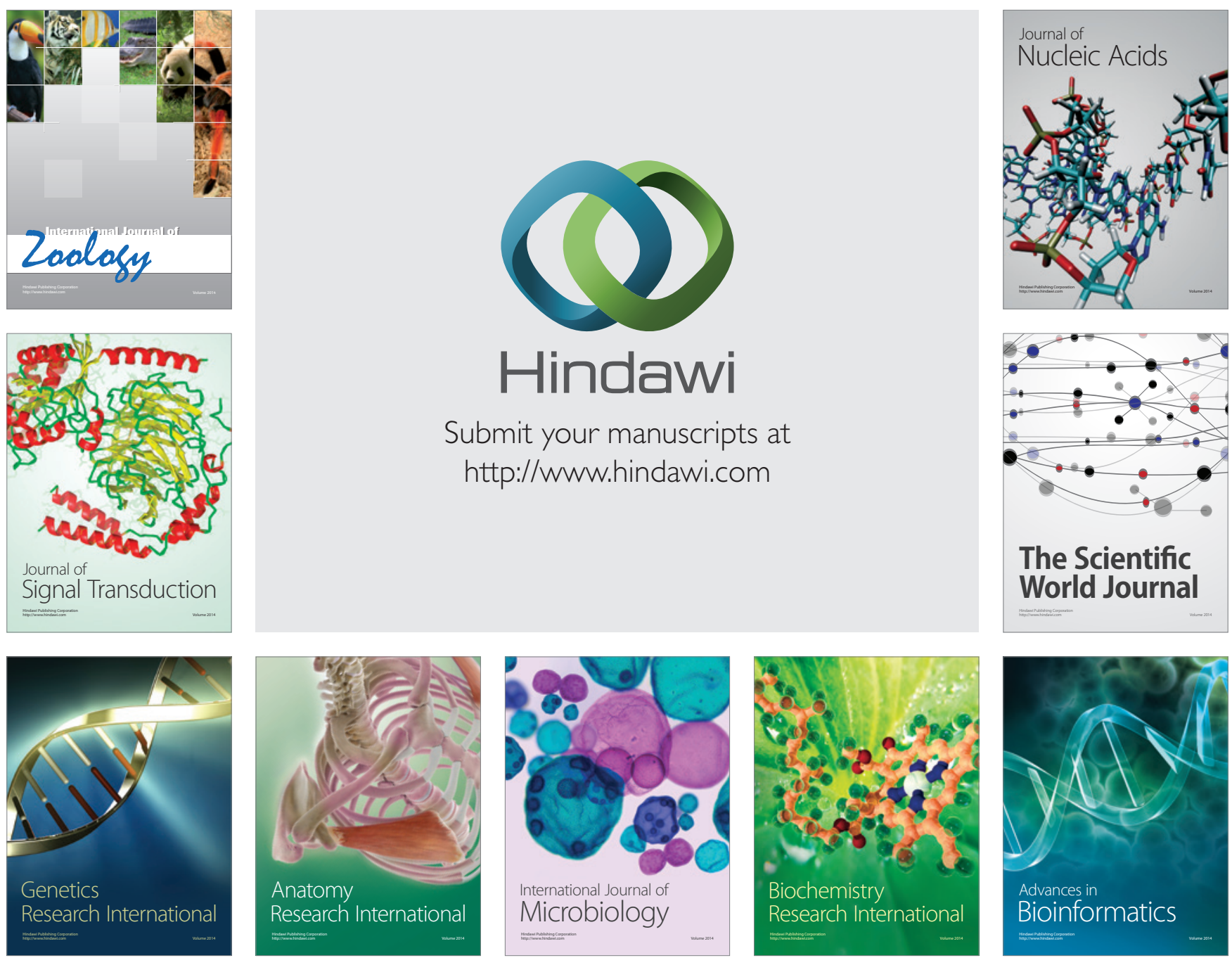

The Scientific World Journal
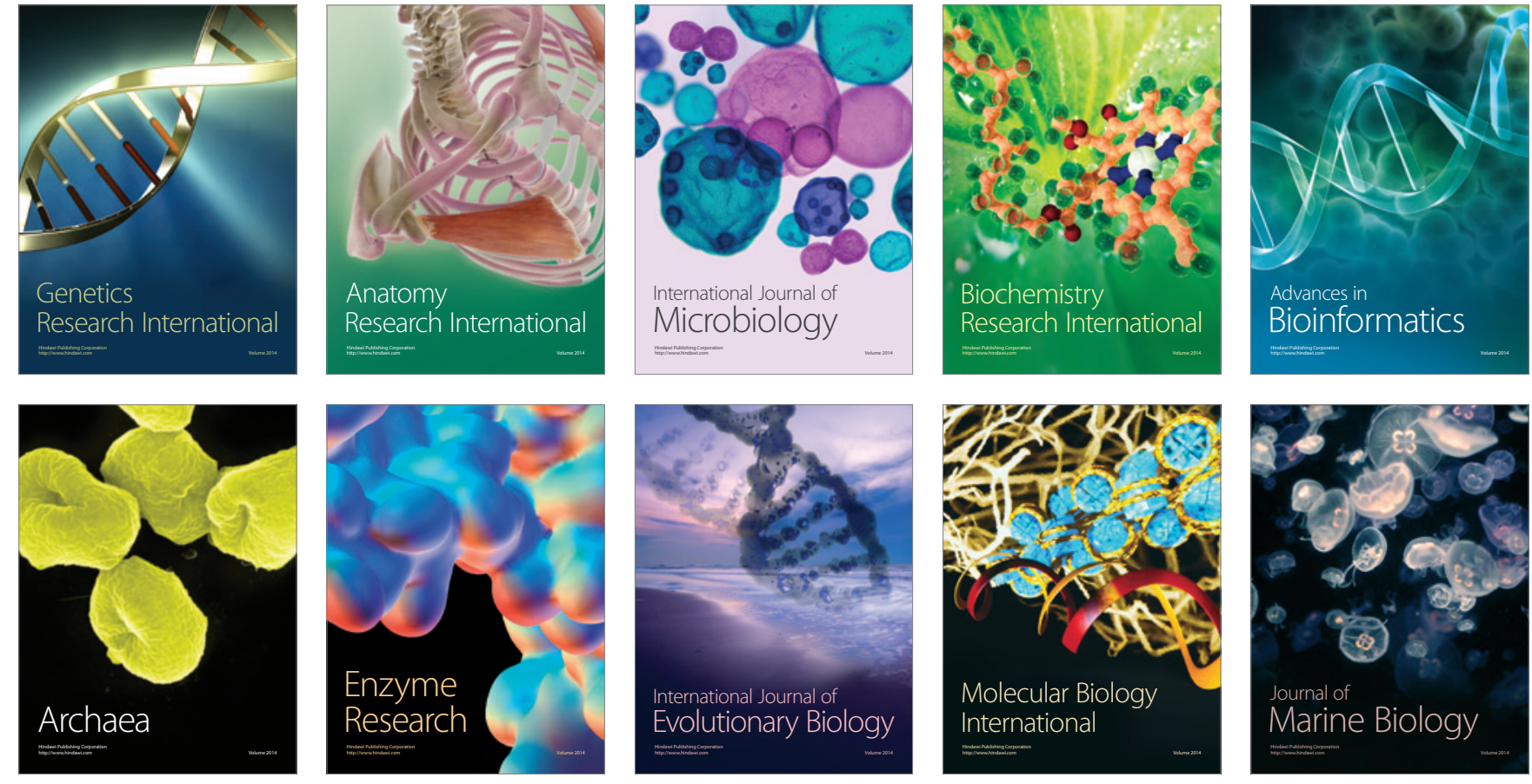